\title{
Potential of chitosan coating in delaying the postharvest anthracnose (Colletotrichum gloeosporioides Penz.) of Eksotika II papaya
}

\begin{abstract}
The in vitro and in vivo fungicidal activity of chitosan was studied against Colletotrichum gloeosporioides, the causal agent of anthracnose in papaya fruits. Chitosan at $1.5 \%$ and $2.0 \%$ concentrations showed a fungistatic effect with $90-100 \%$ inhibition (significant at $\mathrm{P} \leq 0.05$ ) of the fungal mycelial growth. Changes in the conidial morphology were also observed with the higher chitosan concentrations after 7-h incubation. In vivo studies showed that $1.5 \%$ and $2.0 \%$ chitosan coatings on papaya not only controlled the fruit decay but also delayed the onset of disease symptoms by 3-4 weeks during 5 weeks storage at $12 \pm 1{ }^{\circ} \mathrm{C}$ and slowed down the subsequent disease development. However, when leaving the fruits to ripen at ambient temperature $\left(28 \pm 2^{\circ} \mathrm{C}\right), 2.0 \%$ chitosan was less effective than $1.5 \%$ in controlling the disease development. Chitosan coatings also delayed the ripening process by maintaining the firmness levels, soluble solids concentration and titratable acidity values during and after storage.
\end{abstract}

Keyword: Anthracnose, chitosan, disease development, papaya 